\title{
Transport in Nanoporous Materials Including MOFs: The Applicability of Fick's Laws **
}

\author{
Tobias Titze, Alexander Lauerer, Lars Heinke, Christian Chmelik, Nils E. R. Zimmermann, Frerich J. \\ Keil, Douglas M. Ruthven, and Jörg Kärger*
}

\begin{abstract}
Diffusion in nanoporous host-guest systems is often considered to be too complicated to comply with such "simple" relations as Fick's first and second laws of diffusion. However, we show here that the microscopic techniques of diffusion measurement, notably the pulsed field gradient (PFG) technique of NMR and microimaging by interference microscopy (IFM) and IR microscopy (IRM), provide direct experimental evidence of the applicability of Fick's laws to such systems. This remains true in many situations, even when the detailed mechanism is complex. The limitations of the diffusion model are also discussed with reference to the extensive literature of this subject.
\end{abstract}

Thermal energy gives rise to permanent irregular molecular motion. In systems of non-homogeneously distributed molecules, this irregular movement results in fluxes that are correlated with the gradients in molecular concentration by Fick's $1^{\text {st }}$ law:

$j=-D \frac{\partial c}{\partial x}$

Combined with the law of matter conservation, the "continuity relation" $\partial c / \partial t=-\partial j / \partial x$, Eq. (1) yields Fick's $2^{\text {nd }}$ law:

$\frac{\partial c}{\partial t}=\frac{\partial}{\partial x}\left(D \frac{\partial c}{\partial x}\right)$

If the diffusivity $D$ is independent of concentration, Eq. (2) simplifies to:

$\frac{\partial c}{\partial t}=D \frac{\partial^{2} c}{\partial x^{2}}$.

[*] T. Titze, A. Lauerer, Dr. C. Chmelik, Prof. J. Kärger

Department of Interface Physics, University of Leipzig

Linnéstrasse 5, 04103 Leipzig (Germany)

E-mail: kaerger@physik.uni-leipzig.de

Dr. L. Heinke

Institute of Functional Interfaces, Karlsruher Institut für Technologie

Kaiserstraße 12, 76131 Karlsruhe (Germany)

Dr. N. E. R. Zimmermann

Computational Research Division, Lawrence Berkeley National

Laboratory

1 Cyclotron Road, Berkeley, CA 94720 (USA)

Prof. F. J. Keil

Department of Chemical Reaction Engineering, Hamburg University of Technology

Eissendorfer Strasse 38, 21073 Hamburg (Germany)

Prof. D. M. Ruthven

Chemical and Biological Engineering, University of Maine

5737 Jenness Hall, Orono, ME 04469 (USA)

${ }^{\left.{ }^{\star *}\right] \quad F i n a n c i a l ~ s u p p o r t ~ b y ~ t h e ~ G e r m a n ~ S c i e n c e ~ F o u n d a t i o n ~(D F G), ~ F o n d s ~}$ der Chemischen Industrie and Alexander von Humboldt Foundation (grant for D. M. R.'s stay in Leipzig as an AvH Research Awardee) is gratefully acknowledged.

Supporting information for this article is available on the WWW under http://dx.doi.org/10.1002/anie.2015xxxxx
In particular the latter relation holds for the migration of labelled molecules within unlabeled surroundings under equilibrium conditions, commonly referred to as self-diffusion. This is the situation in PFG NMR diffusion studies. ${ }^{[1,2]}$

Relations between physical quantities can only be meaningful if the quantities themselves are meaningfully defined. The fluxes and concentrations as the relevant quantities in Eqs. (1) - (3) must, therefore, be based on unit areas and unit volumes which are large enough so that the fluxes and concentrations become homogeneous functions of the location $x$. As a consequence unit volumes and areas must notably exceed the pore size (just as, in bulk fluids, they must exceed the size of the molecules). On the other hand, differential equations such as Eqs. (1) - (3) are only meaningful if unit volumes and areas are small relative to the size of the system under study. With pore diameters typically of nanometers and particle/crystal sizes of micrometers, both requirements are generally easily fulfilled.

The concept formulated by the diffusion equations (1) - (3) has been applied to mass transfer in nanoporous materials, even from the earliest studies of adsorption and catalysis. Examples include Damköhler's pioneering papers ${ }^{[3,4]}$ and the classic textbooks by Satterfield and Sherwood ${ }^{[5]}$ and Petersen. ${ }^{[6]}$ The diffusivities were well defined in the context of Eqs. (1) - (3) but their accessibility by direct measurement was limited because, at that time, the guest concentrations and guest fluxes appearing in these equations were generally not amenable to direct observation. The diffusivities were therefore derived from observations of "macroscopic" phenomena controlled by diffusion. Examples include measurements of the rates of molecular uptake and release by beds or aggregates of nanoporous materials and heterogeneously catalyzed reactions.

Theoretical models were developed to relate the measured diffusivities to more fundamental parameters such as the molecular density of the adsorbed phase, the pore diameter, the tortuosity of the pore network and the corresponding gas or liquid phase diffusivities. These approaches proved to be quite successful yielding, in many cases, good agreement with the macroscopic measurements, especially when the pores are large relative to the molecular diameter of the sorbate molecules. ${ }^{[7-9]}$

This approach breaks down when the pore diameter is close to the molecular diameter (the configurational diffusion regime) since the diffusivity is then determined mainly by repulsive interactions with the pore wall. Although in this regime the diffusivities cannot be related directly to other transport properties they remain perfectly valid as fundamental parameters characterizing the migration rate.

It is only relatively recently, with the development of microscopic techniques for diffusion measurement, that it has become possible to investigate directly by experiment whether or not mass transfer in a given nanoporous host-guest system follows the laws of ordinary diffusion as given by Eqs. (1) - (3). These techniques, notably the pulsed field gradient (PFG) 
technique of NMR and micro-imaging by interference microscopy and IR microscopy, cover diffusion paths from hundreds of nanometers to hundreds of micrometers as required for diffusion measurement in nanoporous materials. ${ }^{[10]}$ In contrast to the single-molecule techniques ${ }^{[11]}$ they collect, by their very nature, information about ensembles of molecules (from typically $10^{10}$ up to even much larger numbers). Recording data for many molecules is a prerequisite for meaningful application of Eqs. (1) - (3).

To rationalize the evidence that PFG NMR is able to provide in favor of the Fick's laws, we recall that the primary quantity accessible by PFG NMR, namely the attenuation of the NMR signal as a function of the intensity of the applied field gradient pulses, is the Fourier transform of the (mean) propagator. The propagator is defined as the probability distribution $c^{*}(x, t)$ of an ensemble of labelled molecules (within an unlabeled, homogeneously distributed molecular entity) at time $t$, when at time $t=0$ all these molecules have been positioned at $x=0$. For a system following Eq. (3), $c^{*}(x, t)$ is given by the Gaussian

$c^{*}(x, t)=\frac{1}{\sqrt{4 \pi D t}} \exp \left(-\frac{x^{2}}{4 D t}\right)$.

It follows that, if the PFG NMR data reveal a propagator of this form, the diffusion process must be Fickian (i.e in accordance with Eqs. 1 - 3).

Examples for which exactly this behavior has been observed include purely microporous and mesoporous zeolites ${ }^{[12]}$, activated carbon $^{[13]}$, nanoporous glass ${ }^{[14]}$ and ordered mesoporous silica of type SBA-15. ${ }^{[15]}$ The validity of the Fickian model and the physical meaning of the diffusivity appearing in Eq. (3) do not depend on the detailed mechanism of molecular movement. Exploration of the individual steps and the mechanisms that contribute to the diffusion process, in continuation of the work of Damköhler and his successors ${ }^{[4-9,16]}$, remains a challenging task of current research. ${ }^{[17,18-22]}$

Under non-equilibrium conditions (i.e. during uptake and release) concentrations vary with time and space. The recent advent of microimaging ${ }^{[23,24,25]}$ has made it possible to record the evolution of such profiles with unprecedented accuracy. Figure 1a shows the concentration profiles of cyclohexane, determined in this way during uptake by a porous glass. The host material was recently applied for an in-situ study of the hydrogenation of benzene to cyclohexane, where one may also find a detailed description of the material. ${ }^{[26]}$ To reproduce the profiles by a solution of the diffusion equation with the appropriate initial and boundary conditions one must allow for the possibility that the diffusivity may be concentration dependent (in contrast to the situation in self- or tracer diffusion measurements by PFG NMR). The analysis must therefore be based on Eq. (2) rather than Eq. (3). The results of this analysis (see Ref. ${ }^{27}$ for details) are shown in Figure 1a by the full lines. The concentration dependence of the diffusivity used for this fit is shown in Figure 1b. The dramatic decrease in diffusivity at concentrations approaching zero suggests the existence of a small number of strong adsorption sites, while a distinct increase in the diffusivities at intermediate concentrations is quite common for diffusion in nanoporous materials under non-equilibrium conditions (i.e. for transport or Fickian diffusivities). It is caused by the "thermodynamic factor" $d \ln c(p) / d \ln p$ which gives rise to a driving force for diffusive fluxes under non-equilibrium conditions $(\approx$ $1 /(1-\theta)$ for Langmuir-type isotherms) acting in addition to mere thermal motion. ${ }^{[2,25,28]}$ Self-consistency of the results in terms of Fick's 2nd law is revealed by the Boltzmann-Matano plot $^{[29]}$ shown in Figure 1c. In such diagrams, all concentration profiles are plotted together as functions of $x / \sqrt{t}$. If transport resistances at the crystal surface are negligible, the profiles collapse onto a single trend line, as seen in Figure 1c.
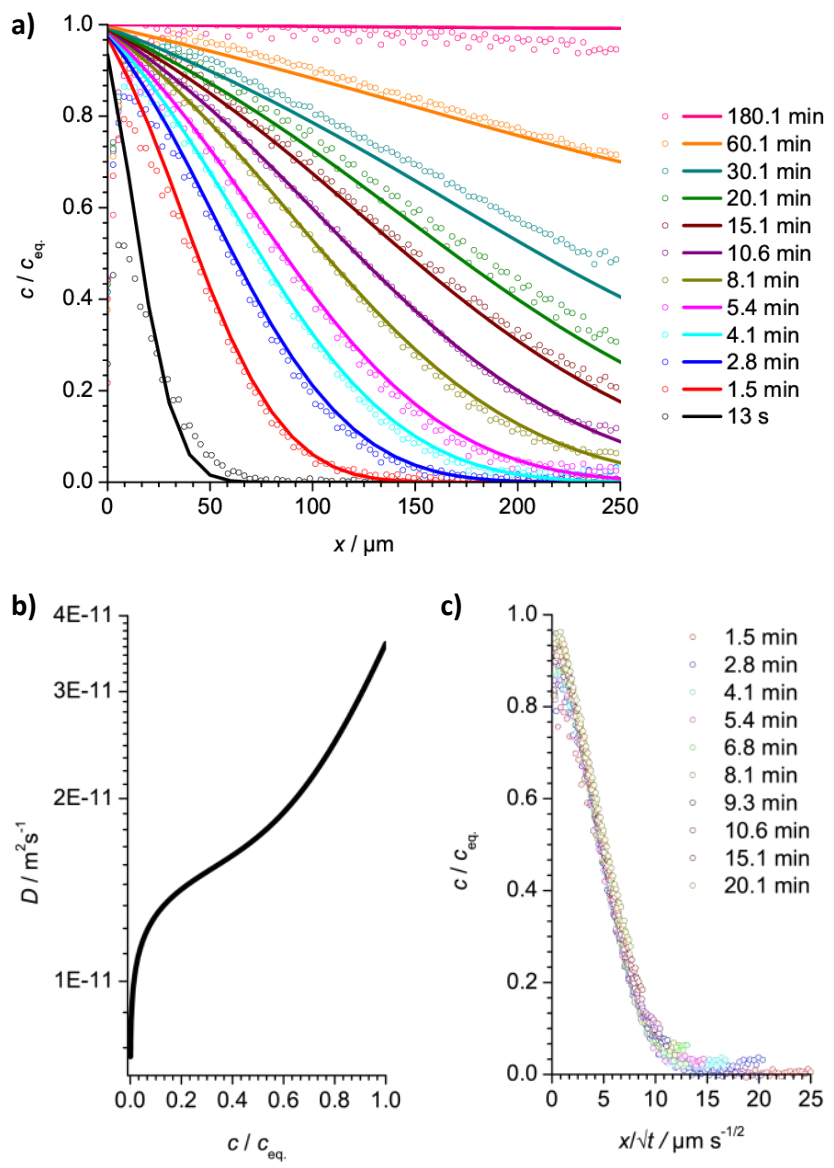

Figure 1. (a) Transient concentration profiles during molecular uptake of cyclohexane by nanoporous glass induced by a pressure step from 0 to $0.1 \mathrm{mbar}$ as recorded by IRM (open circles) at $298 \mathrm{~K}$. The full lines correspond to their prediction by the respective solutions of Fick's $2^{\text {nd }}$ law (Eq. (2)) with the respective initial and boundary conditions and with the concentration dependency of the diffusivity as given in (b). Compatibility with Fick's diffusion laws is also documented by the Boltzmann-Matano-plot in (c) where intraparticle concentrations are shown to be a unique function of $x / \sqrt{t}$.

Mass transfer in nanoporous materials cannot always be assumed to follow the "simple" laws of ordinary diffusion. Deviations from Fickian behavior are to be expected if, during the observation time, there is no fast exchange between states of different mobility. Such behavior may be encountered in hierarchical pore systems with limited exchange between the micro- and meso-/macro-pores. ${ }^{[30]} \mathrm{A}$ similar situation may arise in heterogeneous adsorbents with a wide range of site energies. The behavior of such systems may often be represented by the diffusion-immobilization (diffusion-reaction) model. The general features of this model were discussed in some detail many years ago in Crank's classic book. ${ }^{[31]} \mathrm{A}$ wide range of different patterns of behavior is predicted depending on the ratio of the diffusional 
time constant for the mobile phase $\left(D / R^{2}\right)$ and the rate constant for immobilization (or adsorption). In the limiting case of rapid equilibration the behavior follows the Fickian model with a diffusivity given by $D /(1+K)$ where $D$ is the diffusivity of the mobile phase and $K$ is the adsorption equilibrium constant.

A model of this type was used by Sherry to account for the kinetics of isotopic ion exchange in zeolite $X .{ }^{\left[{ }^{32]}\right.} A$ more recent example is provided by the data of Grzybowski and coworkers ${ }^{[33,34]}$ for the uptake of pyronin B in MOF-5. Although not originally analyzed in this way the transient concentration profiles from that study are accurately represented by the Fickian diffusion model with a constant diffusivity, as shown in Figure 2. This implies rapid equilibration with linear equilibrium between the mobile and adsorbed molecules. Details are given in the Supplementary Information. This result is in agreement with numerous, experimental and computational, studies of the adsorption of guest molecules in MOF-type host systems ${ }^{[18-}$ $22,24,25,35]$ where mass transfer has been observed to follow the normal diffusion model. However, the diffusion-immobilization model implies that simple Fickian behavior will be observed only if the immobilization reaction is much faster than the diffusion of the mobile molecules. When the rates of diffusion and immobilization are comparable deviations from Fickian behavior are to be expected. The diffusion-immobilization model therefore provides a possible explanation of the non-Fickian behavior observed in a number of studies - for example Ref. ${ }^{36}$.

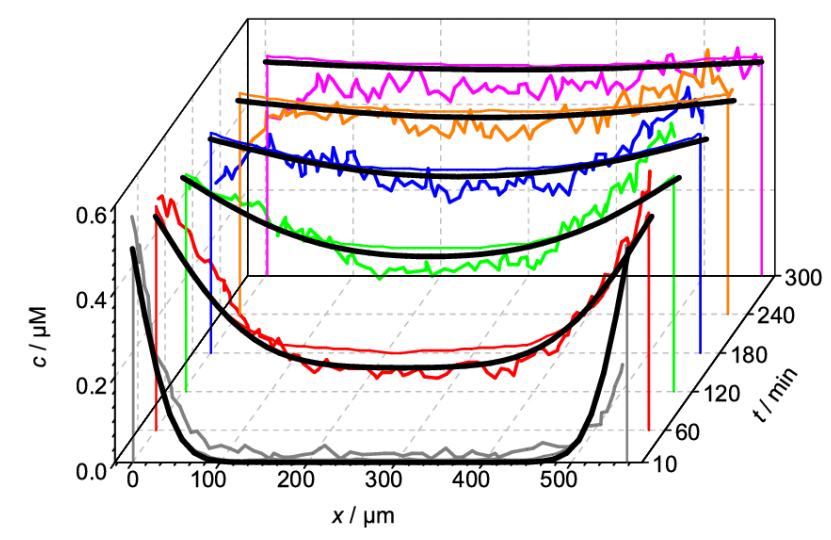

Figure 2. Concentration profiles of pyronin $B(P B)$ upon uptake by nanoporous crystals of type MOF-5, recorded by confocal laser scanning microscopy (experimental data of Fig. 3d of Ref. ${ }^{34}$ ) and analytical approaches to these data given in Ref. ${ }^{34}$ (thin smooth curves) resulting with the "reaction-diffusion" equation in Ref. ${ }^{34}$ and by solution of Fick's laws (thick black curves).

Fick's $1^{\text {st }}$ and $2^{\text {nd }}$ laws have been shown to provide us with excellent tools for the quantitative study of diffusional mass transfer in nanoporous materials. Today, experimental techniques are powerful enough to allow compatibility checks in numerous cases. Both the experimental evidence and molecular modelling verify that, despite its intrinsic complexity, mass transfer in nanoporous materials can often be described almost exactly by the Fickian diffusion laws. The relevant rate parameter which, by its use in the Fick equations, fits precisely the formal definition of a "diffusivity" should obviously be called a diffusivity!
Keywords: diffusion • Fick's laws • nanoporous materials • zeolites • MOFs

[1] W. S. Price, NMR Studies of Translational Motion, University Press Cambridge, 2009.

[2] J. Kärger, D. M. Ruthven, D. N. Theodorou, Diffusion in nanoporous materials, Wiley - VCH, Weinheim, 2012.

[3] G. Damköhler, Der Chemie-Ingenieur 1937, 3, 359-488.

[4] G. Damköhler, International Chemical Engineering 1988, 28, 132-198.

[5] C. N. Satterfield, T. K. Sherwood, The Role of Diffusion inCatalysis, Addison-Wesley, Reading, MA, 1963.

[6] E. E. Petersen, Chemical Reaction Analysis, Prentice-Hall, Englewood Cliffs, N.J., 1965

[7] C. H. Amberg, E. Echigoya, Can. J. Chem. Eng. 1961, 39, 215-218.

[8] N. Wakao, J. M. Smith, Chem. Eng. Sci. 1962, 17, 825-834.

[9] P. B. Weisz, A. B. Schwartz, J. Catal. 1962, 1, 399-406.

[10] J. Kärger, ChemPhysChem 2015, 16, 24-51.

[11] a) C. Bräuchle, D. C. Lamb, J. Michaelis (Eds.) Single particle tracking and single molecule energy transfer, Wiley- $\mathrm{VCH}$, Weinheim, 2010; b) B. M. Weckhuysen, Angew. Chem. Int. Ed. 2009, 48, 4910-4943.

[12] a) P. Kortunov, S. Vasenkov, J. Kärger, M. F. Elia, M. Perez, M. Stöcker, G. K. Papadopoulos, D. Theodorou, B. Drescher, G. McElhiney et al., Chem. Mat. 2005, 17, 2466-2474; b) P. Kortunov, S. Vasenkov, J. Kärger, R. Valiullin, P. Gottschalk, M. F. Elia, M. Perez, M. Stöcker, B. Drescher, G. McElhiney et al., J. Am. Chem. Soc. 2005, 127, 1305513059 ; c) Z. Adem, F. Guenneau, M. A. Springuel-Huet, A. Gedeon, Micropor. Mesopor. Mater. 2008, 114, 337-342; d) A. Menjoge, S. A. Bradley, D. B. Galloway, J. J. Low, S. Prabhakar, S. Vasenkov, Micro. Mesopor. Mater. 2010, 135, 30-36; e) D. Mehlhorn, R. Valiullin, J. Kärger, K. Cho, R. Ryoo, Micro. Mesopor. Mater. 2012, 164, 273-279; f) D. Mehlhorn, R. Valiullin, J. Kärger, K. Cho, R. Ryoo, Chem. Phys. Chem. 2012, 13, 1495-1499.

[13] a) J. Kärger, J. Caro, P. Cool, M. O. Coppens, D. Jones, F. Kapteijn, F. Rodríguez-Reinoso, M. Stocker, D. Theodorou, E. F. Vansant et al., Chem. Eng. Technol. 2009, 32, 1494-1511; b) F. Furtado, P. Galvosas, M. Gonçalvezd, F. D. Kopinke, S. Naumov, F. Rodríguez-Reinoso, U. Roland, R. Valiullin, J. Kärger, Microporous and Mesoporous Materials 2011, 141, 184-191.

[14] a) C. Chmelik, D. Enke, P. Galvosas, O. C. Gobin, A. Jentys, H. Jobic, J. Kärger, C. Krause, J. Kullmann, J. A. Lercher et al., ChemPhysChem 2011, 12, 1130-1134; b) F. Feil, S. Naumov, J. Michaelis, R. Valiullin, D. Enke, J. Kärger, C. Bräuchle, Angew. Chem. Int. Ed. 2012, 51, 1152-1155.

[15] a) S. Naumov, R. Valiullin, J. Kärger, R. Pitchumani, M. O. Coppens, Microporous Mesoporous Mater. 2008, 110, 37-40; b) V. Meynen, P. Cool, E. F. Vansant, P. Kortunov, F. Grinberg, J. Kärger, M. Mertens, O. I. Lebedev, G. van Tendeloo, Microporous Mesoporous Mat. 2007, 99 , 14-22.

[16] a) D. D. Do, Adsorption Analysis: Equilibria and Kinetics, Imperial College Press, London, 1998; b) E. L. Cussler, Diffusion: Mass Transfer in Fluid Systems, Cambridge University Press, Cambridge, 2009; c) G. F. Froment, K. B. Bischoff, J. de Wilde, Chemical Reactor Analysis and Design, John Wiley \& Sons, 2011.

[17] a) P. Demontis, L. A. Fenu, G. B. Suffritti, J. Phys. Chem. B 2005, 109, 18081-18087; b) E. Yoda, J. N. Kondo, K. Domen, J. Phys. Chem. B 2005, 109, 1464-1472; c) M. E. Cordero, R. Natividad, L. G. Zárate, J. A. Hernandez-Servin, J. Salas, Catalysis Today 2014, 220-222, 113123; d) N. E. R. Zimmermann, T. J. Zabel, F. J. Keil, J. Phys. Chem. C 2013, 117, 7384-7390.

[18] N. Rosenbach, H. Jobic, A. Ghoufi, F. Salles, G. Maurin, S. Bourrelly, P. L. Llewellyn, T. Devic, C. Serre, G. Ferey, Angew. Chem. Int. Ed. 2008 47, 6611-6615.

[19] F. Salles, H. Jobic, G. Maurin, M. M. Koza, P. L. Llewellyn, T. Devic, C. Serre, G. Férey, Phys. Rev. Lett. 2008, 100, 245901.

[20] F. Salles, H. Jobic, A. Ghoufi, P. L. Llewellyn, C. Serre, S. Bourelly, G. Ferey, G. Maurin, Angew. Chem. Int. Ed. 2009, 121, 8485-8489.

[21] D. C. Ford, D. Dubbeldam, R. Q. Snurr, V. Künzel, M. Wehring, F. Stallmach, J. Kärger, U. Müller, J. Phys. Chem. Lett. 2012, 3, 930-933.

[22] N. Rosenbach, H. Jobic, A. Ghoufi, T. Devic, M. M. Koza, N. Ramsahye, C. J. Mota, C. Serre, G. Maurin, J. Phys. Chem. C 2014, 118, 1447114477.

[23] a) J. Kärger, P. Kortunov, S. Vasenkov, L. Heinke, D. B. Shah, R. A Rakoczy, Y. Traa, J. Weitkamp, Angew. Chem.-Int. Edit. 2006, 45, 7846-7849; b) D. Tzoulaki, L. Heinke, W. Schmidt, U. Wilczok, J. Kärger, Angew. Chem., Int. Ed. 2008, 47, 3954-3957.

[24] D. Tzoulaki, L. Heinke, J. Li, H. Lim, D. Olson, J. Caro, R. Krishna, C. Chmelik, J. Kärger, Angew. Chem. Int. Ed. 2009, 48, 3525-3528.

[25] J. Kärger, T. Binder, C. Chmelik, F. Hibbe, H. Krautscheid, R. Krishna, J. Weitkamp, Nat. Mat. 2014, 13, 333-343.

[26] T. Titze, C. Chmelik, J. Kullmann, L. Prager, E. Miersemann, R. Gläser, D. Enke, J. Weitkamp, J. Kärger, Angew. Chem. Int. Ed. 2015, 54, 5060-5064.

[27] L. Heinke, J. Kärger, New Journal of Physics 2008, 10, 23035. 
[28] a) R. Krishna, J. M. van Baten, Phys. Chem. Chem. Phys. 2013, 15, 7994; b) R. Krishna, Micropor. Mesopor. Mater. 2014, 185, 30-50.

[29] a) L. Boltzmann, Wied. Ann. 1894, 53, 959-964; b) C. Matano, Japan. J. Phys. 1933, 8, 109; c) P. Kortunov, L. Heinke, J. Kärger, Chem. Mat. 2007, 19, 3917-3923.

[30] a) M. Hartmann, Angew. Chem. Int. Ed. 2004, 43, 5880-5882; b) M. Rincon Bonilla, T. Titze, F. Schmidt, D. Mehlhorn, C. Chmelik, R. Valiullin, S. K. Bhatia, S. Kaskel, R. Ryoo, J. Kärger, Materials (open access) 2013, 6, 2662-2688; c) L. Gueudré, M. Milina, S. Mitchell, J. Pérez-Ramírez, Adv. Funct. Mater. 2014, 24, 209-219.

[31] J. Crank, Mathematics of Diffusion, Oxford, 1956.

[32] H. S. Sherry, Adv. Chem. 1971, 101, 350-379.

[33] S. Soh, M. Byrska, K. Kandere-Grzybowska, B. A. Grzybowski, Angew. Chem. Int. Ed. 2010, 49, 4170-4198.
[34] S. Han, T. M. Hermans, P. E. Fuller, Y. Wei, B. A. Grzybowski, Angew. Chem. Int. Ed. 2012, 51, 2662-2666.

[35] a) C. Chmelik, L. Heinke, P. Kortunov, J. Li, D. Olson, D. Tzoulaki, J. Weitkamp, J. Kärger, Chem. Phys. Chem. 2009, 10, 2623-2627; b) C. Chmelik, H. Bux, J. Caro, L. Heinke, F. Hibbe, T. Titze, J. Kärger, Phys. Rev. Lett. 2010, 104, 85902; c) L. Heinke, Z. Gu, C. Wöll, Nat. Comms. 2014, 5, 4562

[36] S. Han, Y. Wei, C. Valente, I. Lagzi, J. J. Gassensmith, A. Coskun, J. F. Stoddart, B. A. Grzybowski, Journal of the American Chemical Society 2010, 132, 16358-16361. 


\section{Entry for the Table of Contents}

\section{COMMUNICATION}

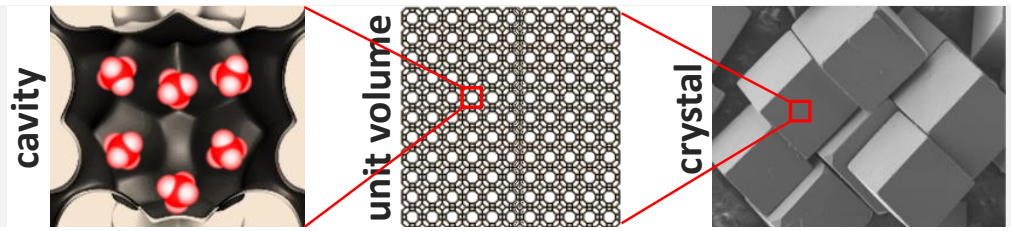

No more Diffusion confusion: Although the elementary steps of molecular propagation in nanoporous materials may be quite intricate, overall mass transfer in the individual particles generally follows the simple Fickian laws of diffusion. Even in a complex system, the diffusion coefficient is a well-defined quantity and the addition of qualifiers such as "effective", "seeming", or "apparent" is unnecessary and even misleading.
Transport in Nanoporous Materials Including MOFs: The Applicability of Fick's Laws

Tobias Titze, Alexander Lauerer, Dr. Lars Heinke, Christian Chmelik, Nils E. R. Zimmermann, Frerich J. Keil, Douglas M. Ruthven, and Jörg Kärger*

Angew. Chem. Int. Ed. 54 (2015), 14580 - Page 14583,

DOI: 10.1002/anie.201506954 . 
Supporting Information to accompany the article:

\title{
Transport in Nanoporous Materials Including MOFs: The Applicability of Fick's Laws
}

\author{
Tobias Titze ${ }^{1}$, Alexander Lauerer ${ }^{1}$, Lars Heinke ${ }^{2}$, Christian Chmelik ${ }^{1}$, Nils E. R. Zimmermann ${ }^{3}$, \\ Frerich J. Keil' ${ }^{4}$, Douglas M. Ruthven ${ }^{5}$, and Jörg Kärger ${ }^{1 *}$
}

${ }^{1}$ Department of Interface Physics, University of Leipzig, Linnéstrasse 5, 04103 Leipzig (Germany);

${ }^{2}$ Institute of Functional Interfaces, Karlsruher Institut für Technologie, Kaiserstraße 12, 76131 Karlsruhe (Germany)

${ }^{3}$ Dr. N. E. R. Zimmermann, Computational Research Division, Lawrence Berkeley National Laboratory, 1 Cyclotron Road, Berkeley, CA 94720 (USA)

${ }^{4}$ Prof. F. J. Keil, Department of Chemical Reaction Engineering, Hamburg University of Technology, Eissendorfer Strasse 38, 21073 Hamburg (Germany)

${ }^{5}$ Prof. D. M. Ruthven, Chemical and Biological Engineering, University of Maine, 5737 Jenness Hall, Orono, ME 04469 (USA)

*Corresponding author; E-mail address: kaerger@physik.uni-leipzig.de (J. Kärger) 


\section{Solution of Fick's $2^{\text {nd }}$ law fitted to the experimental concentration data reported by Han et al. ${ }^{[1]}$}

The evolution of concentration $c(x, y, z, t)$ upon molecular uptake or release within in a cuboid of extension $2 l_{i}$ in direction $i$ with $i=x, y, z$ is given by the relation:

$$
\begin{aligned}
& \frac{c(t)-c_{0}}{c_{\infty}-c_{0}}=1-8 L_{x} L_{y} L_{z} \\
& \quad \cdot \sum_{n=1}^{\infty} \sum_{m=1}^{\infty} \sum_{k=1}^{\infty} \frac{\cos \frac{\beta_{n} x}{l_{x}} \cos \frac{\beta_{m} y}{l_{y}} \cos \frac{\beta_{k} z}{l_{z}} \exp \left(-D t\left(\frac{\beta_{n}^{2}}{l_{x}^{2}}+\frac{\beta_{m}^{2}}{l_{y}^{2}}+\frac{\beta_{k}^{2}}{l_{z}^{2}}\right)\right)}{\left(\beta_{n}^{2}+L_{x}^{2}+L_{x}\right)\left(\beta_{m}^{2}+L_{y}^{2}+L_{y}\right)\left(\beta_{k}^{2}+L_{z}^{2}+L_{z}\right) \cos \beta_{n} \cos \beta_{m} \cos \beta_{k}}
\end{aligned}
$$

with $L_{i}=l_{i} \alpha / D$ and $\beta_{j}$ denoting the positive roots of $\beta_{j} \tan \beta_{j}=L_{i}$. Both the diffusivity $D$ and the surface permeability $\alpha$ are, in this relation, assumed to be independent of concentration, with diffusion considered to be isotropic. Eq. (S1) results as the general solution $F(x, y, z, t)$ which, under such conditions, is known to be simply the product of the orthogonal solutions $F_{x}(x, t), F_{y}(y, t)$ and $F_{z}(z, t):[2,3]$

$$
F(x, y, z, t)=F_{x}(x, t) \cdot F_{y}(y, t) \cdot F_{z}(z, t)
$$

From textbooks (see p. $45 \mathrm{in}^{[2]}$ or p. $152 \mathrm{in}^{[4]}$ ), the individual, orthogonal solutions (uptake by parallelsided slab) are known to be:

$$
F_{x}(x, t)=c(x, t)=1-\sum_{n=1}^{\infty} \frac{2 L_{x} \cos \frac{\beta_{n} x}{l_{x}} \exp \left(-\frac{\beta_{n}^{2} D t}{l_{x}^{2}}\right)}{\left(\beta_{n}^{2}+L_{x}^{2}+L_{x}\right) \cos \beta_{n}}
$$

The easiest way of getting, via Eq. (S2), from Eq. (S3) to Eq. (S1) is by considering the evolution of concentrations during molecular release rather than during uptake, making use of the identities $c_{\text {release }}(x, t) \equiv 1-c(x, t)$ and $c_{\text {release }}(x, y, z, t) \equiv 1-c(x, y, z, t)$.

The plots (thick black lines) shown in Fig. 2 result as the best fits of Eq. (S1) to the experimental data of Ref. ${ }^{[1]}$ with $y=z=0$ (center of crystal) and the $x$ coordinate directed along the profiles shown in Figs. 3d of Ref. ${ }^{[1]}$ and in Fig. 2 in the main text of the present communication, respectively. With crystal extensions of $L_{x}=L_{y}=565 \mu \mathrm{m}$ and $L_{z}=250 \mu \mathrm{m}$, the best fit between Eq. (S1) and the experimental data was attained with $D=1.02 \cdot 10^{-12} \mathrm{~m}^{2} \mathrm{~s}^{-1}$ and negligible surface resistances (i.e. $L_{i}=l_{i} \alpha / D \gg 1$ ). The resulting curves are shown in Fig. 2 . 


\section{References}

[1] S. Han, T. M. Hermans, P. E. Fuller, Y. Wei, B. A. Grzybowski, Angew. Chem. Int. Ed. 2012, 51, 2662-2666.

[2] J. Crank, The Mathematics of Diffusion, Clarendon Press, Oxford, 1975.

[3] H. Tautz, Wärmeleitung und Temperaturausgleich, Akademieverlag, Berlin, 1971.

[4] J. Kärger, D. M. Ruthven, D. N. Theodorou, Diffusion in nanoporous materials, Wiley - VCH, Weinheim, 2012. 